\title{
SOLUTIONS OF CONVEX BETHE ANSATZ EQUATIONS AND THE ZEROS OF (BASIC) HYPERGEOMETRIC ORTHOGONAL POLYNOMIALS
}

\author{
J.F. VAN DIEJEN AND E. EMSIZ
}

\begin{abstract}
Via the solutions of systems of algebraic equations of Bethe Ansatz type, we arrive at bounds for the zeros of orthogonal (basic) hypergeometric polynomials belonging to the Askey-Wilson, Wilson and continuous Hahn families.
\end{abstract}

\section{INTRODUCTION}

The study of the zeros of orthogonal polynomials has a rich history [S75] stimulated, in particular, by its relevance for the theory of numerical approximation G04. Of special interest are the zeros of the classical families of hypergeometric orthogonal polynomials, which have been fruitfully analyzed e.g. through SturmLiouville theory and via Stieltjes' electrostatic interpretation S75, ALM82, FR86, G87, ELR94, G98, I00, MMM07, JT09, DJ12, ADGR12, ADGP13, S16. In this work we are mainly concerned with estimates for the locations of the zeros of some well-studied (basic) hypergeometric orthogonal polynomial families belonging to the $(q-)$ Askey scheme AW85, KLS10, while other relevant issues concerning these zeros, such as e.g. their dependence on the parameters S75, M93, I05, interlacing properties S75, D09, HV12, GJRS16, or their asymptotical behavior S75, S10, DHK10, will not be addressed.

A classic bound for the locations of the zeros

$$
\pi>\xi_{1}^{(n)}>\xi_{2}^{(n)} \cdots>\xi_{n-1}^{(n)}>\xi_{n}^{(n)}>0
$$

of the Jacobi polynomial $P_{n}^{(\alpha, \beta)}(\cos (\xi))$ [KLS10, Ch. 9.8] with $-\frac{1}{2} \leq \alpha, \beta \leq \frac{1}{2}$ is provided by Buell's estimate [S75, Eqs. (6.3.5), (6.3.7)]:

$$
\frac{\left(n+1-j+\frac{1}{2}(\alpha+\beta-1)\right) \pi}{n+\frac{1}{2}(\alpha+\beta+1)} \leq \xi_{j}^{(n)} \leq \frac{(n+1-j) \pi}{n+\frac{1}{2}(\alpha+\beta+1)} \quad(j=1, \ldots, n)
$$

(where all inequalities are actually strict unless $\alpha^{2}=\beta^{2}=\frac{1}{4}$ ). For $\alpha=\beta=\frac{1}{2}$ the estimate in Eq. (1.2) becomes exact; indeed, the Jacobi polynomial $P_{n}^{(\alpha, \beta)}(\cos (\xi))$ degenerates in this situation to the Chebyshev polynomial of the second kind $U_{n}(\cos (\xi))=\frac{\sin ((n+1) \xi)}{\sin (\xi)}$.

Date: May 2018.

2010 Mathematics Subject Classification. Primary: 33D45; Secondary 26C10, 33C45, 81R12, $82 \mathrm{~B} 23$.

Key words and phrases. (basic) hypergeometric orthogonal polynomials, zeros of orthogonal polynomials, convex Bethe Ansatz equations.

This work was supported in part by the Fondo Nacional de Desarrollo Cientifico y Tecnológico (FONDECYT) Grants \# 1170179 and \# 1181046. 
Below we will derive a similar estimate for the corresponding zeros of the AskeyWilson polynomial $p_{n}(\cos (\xi) ; a, b, c, d ; q)$ [AW85, [KLS10, Ch. 14.1] with parameters in the domain $-1<a, b, c, d, q<1$ :

$$
\frac{(n+1-j) \pi}{k_{-}^{(n)}(a, b, c, d ; q)} \leq \xi_{j}^{(n)} \leq \frac{(n+1-j) \pi}{k_{+}^{(n)}(a, b, c, d ; q)} \quad(j=1, \ldots, n)
$$

where

$$
\begin{aligned}
& k_{ \pm}^{(n)}(a, b, c, d ; q)=(n-1)\left(\frac{1-|q|}{1+|q|}\right)^{ \pm 1}+ \\
& \quad \frac{1}{2}\left(\left(\frac{1-|a|}{1+|a|}\right)^{ \pm 1}+\left(\frac{1-|b|}{1+|b|}\right)^{ \pm 1}+\left(\frac{1-|c|}{1+|c|}\right)^{ \pm 1}+\left(\frac{1-|d|}{1+|d|}\right)^{ \pm 1}\right) .
\end{aligned}
$$

The estimate in Eqs. (1.3a), (1.3b) becomes exact for vanishing parameters $a, b, c, d$ and $q$, and again $p_{n}(\cos (\xi) ; 0,0,0,0 ; 0)=U_{n}(\cos (\xi))$ in this situation. Notice however that our formula renders only a trivial lower bound (viz. zero) if one of the parameters tends to 1 in absolute value, and that - especially for the larger rootsthe upper bound is nontrivial (i.e. smaller than $\pi$ ) only for parameter values sufficiently close to 0. In particular, the Askey-Wilson estimate in Eqs. (1.3a), (1.3b) merely produces trivial bounds on the zeros of the Jacobi polynomial via a direct application of the well-known degeneration formula (cf. e.g. [KLS10, Ch. 14.10]):

$$
\lim _{q \rightarrow 1} c_{n} p_{n}\left(\cos (\xi) ; q^{\frac{\alpha}{2}+\frac{1}{4}}, q^{\frac{\alpha}{2}+\frac{3}{4}},-q^{\frac{\beta}{2}+\frac{1}{4}},-q^{\frac{\beta}{2}+\frac{3}{4}} ; q\right)=P_{n}^{(\alpha, \beta)}(\cos (\xi))
$$

(where - employing standard notations for the $q$-shifted factorials - the normalization factor is given explicitly by $\left.c_{n}:=q^{\left(\frac{\alpha}{2}+\frac{1}{4}\right) n} /\left(q,-q^{\frac{1}{2}(\alpha+\beta+1)},-q^{\frac{1}{2}(\alpha+\beta+2)} ; q\right)_{n}\right)$.

Many fundamental properties of the Askey-Wilson polynomials were first presented in the seminal memoir [AW85]. The zeros were investigated at an early stage by L. Chihara (for $q$ integral and $a, b, c, d$ rational) in connection with the (non)existence of certain perfect codes [C87. More recently it was observed that the locations of the zeros under consideration are determined by an algebraic system of Bethe Ansatz equations [ILR04, D05, OS05, BC16] stemming from the celebrated second-order $q$-difference equation satisfied by the Askey-Wilson polynomials I05, Ch. 16.5]. Remarkably, this algebraic system turns out to be closely related to a family of Bethe Ansatz equations that emerge when diagonalizing $q$-boson models with open-end boundary interactions LW12, DE17, DEZ18.

We will consider two ample classes of (generalized) Bethe Ansatz type equations that are referred to as Bethe systems of type A and of type B. For special parameter choices, these Bethe equations of type $\mathrm{A}$ and of type $\mathrm{B}$ arise when diagonalizing quantum integrable particle models with periodic boundary conditions and with open-end boundary conditions, respectively. Both types of systems manifest themselves here in three versions: rational $(r)$, hyperbolic $(h)$ and trigonometric $(t)$. We will solve the corresponding Bethe systems by means of a powerful technique going back to Yang and Yang [YY69, M94, G14, KBI93], which provides the Bethe roots

*Whereas a classical result of Bochner characterizes the Jacobi polynomials as "the most general orthogonal family satisfying a linear homogeneous second-order differential equation", the Askey-Wilson polynomials are known to constitute "the most general orthogonal family satisfying a linear homogeneous second-order $q$-difference equation" GH96]. 
in terms of the global minima of an associated family of strictly convex Morse functions and automatically produces bounds estimating the locations of these roots. The bounds in Eqs. (1.3a), (1.3b) for zeros of the Askey-Wilson polynomials then follow via a parameter-specialization of the trigonometric Bethe systems of type B. Similarly, the corresponding rational Bethe equations of type B give rise to lower bounds for the zeros of the Wilson polynomials W80. Finally, lower bounds for the zeros of the symmetric continuous Hahn polynomials AW82 are retrieved via the rational Bethe systems of type A.

The material is organized as follows. In Sections 2 and 3 the Bethe systems of types A and B are exhibited in their general form and solved using the techniques of Yang and Yang YY69. In Section 4 we specialize the parameters in the Bethe system of type B so as to achieve the bounds for the zeros of the Wilson polynomials (rational version) and the Askey-Wilson polynomials (trigonometric version), respectively. In Section 5, an analogous parameter specialization of the rational Bethe system of type A then entails the lower bounds for the zeros of the symmetric continuous Hahn polynomials.

Note. Throughout it will be implicitly assumed that empty products are equal to 1 and that empty sums are equal to 0 . We will also freely employ standard notations for (basic) hypergeometric series and $(q$-)shifted factorials in accordance with the conventions in Ref. KLS10.

\section{Convex Bethe systems of type A}

The idea of the Bethe Ansatz method is to convert the spectral problem for amenable quantum integrable particle models into an algebraic problem: the spectrum is computed through a complete set of solutions to an auxiliary system of algebraic equations [M94, G14, T83, KBI93. The combinatorics of such Bethe Ansatz solutions was investigated recently in Ref. [KS13. Here we will consider a rather wide class of (in general transcendental) Bethe type equations that are convex in the sense that they can be solved in terms of the critical points of an associated family of strictly convex Morse functions using the approach of Yang and Yang YY69, M94, G14, KBI93. Special instances of the type A systems in this section have appeared in the literature in connection with the spectral problems of exactly solvable quantum particle models with periodic boundary conditions. Specifically, the Bethe Ansatz equations governing the spectral problems of the periodic Lieb-Liniger quantum nonlinear Schrödinger equation LL63, YY69, M94, G14, D93, KBI93 and its lattice discretization due to Izergin and Korepin [IK81, D93] (cf. also [KBI93, Ch. VIII.3]) correspond to rational systems of type A. Trigonometric/hyperbolic systems of type A arise in turn as Bethe Ansatz equations for the $q$-boson model [BB92, BIK98, T06, D06, K13] and for the lattice quantum sine-Gordon equation [KBI93, Ch. VIII.5]. The well-studied periodic Heisenberg XXX and XXZ spin chains also give rise to rational and trigonometric/hyperbolic Bethe Ansatz equations of type A [M94, G14, B82, KBI93, but these spin models correspond to parameter values that do not belong to the convex regime considered here. The results in this section therefore do not apply directly to such models and alternative techniques have been incorporated to analyze the corresponding Bethe Ansatz equations in these situations, cf. e.g. Refs. MTV09] and $\underline{\text { K18}}$ and references therein. 
2.1. Bethe equations. Given $u \in\{r, h, t\}$ and

$$
\mathrm{s}(x)=\mathrm{s}^{(u)}(x):= \begin{cases}\frac{x}{2} & \text { if } u=r, \\ \sinh \left(\frac{x}{2}\right) & \text { if } u=h, \\ \sin \left(\frac{x}{2}\right) & \text { if } u=t,\end{cases}
$$

the Bethe system of type $\mathrm{A}$ is defined by $n$ equations in the variables $\xi_{1}, \ldots, \xi_{n}$ of the form

$$
e^{i \alpha \xi_{j}}=e^{2 \pi i \beta} \prod_{1 \leq k \leq K} \frac{\mathrm{s}\left(i a_{k}+\xi_{j}\right)}{\mathrm{s}\left(i a_{k}-\xi_{j}\right)} \prod_{\substack{1 \leq j^{\prime} \leq n, j^{\prime} \neq j \\ 1 \leq l \leq L}} \frac{\mathrm{s}\left(i b_{l}+\xi_{j}-\xi_{j^{\prime}}\right)}{\mathrm{s}\left(i b_{l}-\xi_{j}+\xi_{j^{\prime}}\right)}
$$

$(j=1, \ldots, n)$. Here $\alpha, \beta, a_{1}, \ldots, a_{K}$ and $b_{1}, \ldots, b_{L}$ refer to a choice of $K+L+2$ parameters. Throughout this section we assume (unless explicitly stated otherwise) that

$$
\alpha \in(0, \infty), \quad \beta \in[0,1), \quad a_{k}, b_{l} \in\left\{\begin{array}{ll|}
(0, \infty) & \text { if } u=r \\
(0, \pi) & \text { if } u=h \\
(0, \infty) \cup((0, \infty)+i \pi) & \text { if } u=t
\end{array}\right.
$$

$(k=1, \ldots, K, l=1, \ldots, L)$. As will be seen shortly, these restrictions on the parameters guarantee that the roots of our Bethe system are governed by a convex (Yang-Yang) Morse function.

2.2. Bethe roots. For any $\mu=\left(\mu_{1}, \ldots, \mu_{n}\right)$ belonging to

$$
\Lambda_{\mathrm{A}}:=\left\{\mu \in \mathbb{Z}^{n} \mid \mu_{1}>\mu_{2}>\cdots>\mu_{n}\right\},
$$

let us define the following Morse function in the variable $\boldsymbol{\xi}=\left(\xi_{1}, \ldots, \xi_{n}\right) \in \mathbb{R}^{n}$ :

$$
\begin{aligned}
V_{\mathrm{A}, \mu}^{(u)}(\boldsymbol{\xi} ; \alpha, \beta):= & \sum_{1 \leq j \leq n}\left(\frac{1}{2} \alpha \xi_{j}^{2}-2 \pi\left(\mu_{j}+\beta\right) \xi_{j}+\sum_{1 \leq k \leq K} \int_{0}^{\xi_{j}} v_{a_{k}}(x) \mathrm{d} x\right) \\
& +\sum_{\substack{1 \leq j<j^{\prime} \leq n \\
1 \leq l \leq L}} \int_{0}^{\xi_{j}-\xi_{j^{\prime}}} v_{b_{l}}(x) \mathrm{d} x,
\end{aligned}
$$

with

$$
v_{a}(x)=v_{a}^{(u)}(x):= \begin{cases}\int_{0}^{x} \frac{2 a}{a^{2}+y^{2}} \mathrm{~d} y=2 \arctan \left(\frac{x}{a}\right) & \text { if } u=r, \\ \int_{0}^{x} \frac{\sin (a)}{\cosh (y)-\cos (a)} \mathrm{d} y & \text { if } u=h, \\ \int_{0}^{x} \frac{\sinh (a)}{\cosh (a)-\cos (y)} \mathrm{d} y & \text { if } u=t .\end{cases}
$$

In the spirit of Yang and Yang YY69, this Morse function is designed in such a way that the equation for its critical points (cf. Eq. (2.8) below) reproduces our Bethe equation (2.2) upon exponentiation (cf. the proof of Proposition 2.1 below). The parameter restrictions in Eq. (2.3) now guarantee that $V_{\mathrm{A}, \mu}^{(u)}(\boldsymbol{\xi} ; \alpha, \beta)$ (2.5) is smooth and that $V_{\mathrm{A}, \mu}^{(u)}(\boldsymbol{\xi} ; \alpha, \beta) \rightarrow \infty$ when $|\boldsymbol{\xi}| \rightarrow \infty$, so the function in question possesses a global minimum in $\mathbb{R}^{n}$. Notice in this connection that the contributions from the integrals in Eq. (2.5) are nonnegative for all $\boldsymbol{\xi} \in \mathbb{R}^{n}$ (because $v_{a}(x)$ (2.6) is odd and increasing in $x$ for the relevant parameter values) and that for $|\boldsymbol{\xi}| \rightarrow \infty$ the nonnegative quadratic terms up front dominate possibly negative contributions stemming from the linear terms. 
The minimum in question is unique by convexity. Indeed, the Hessian

$$
\begin{aligned}
& H_{j, j^{\prime}}^{\mathrm{A}}(\boldsymbol{\xi}):=\partial_{\xi_{j}} \partial_{\xi_{j^{\prime}}} V_{\mathrm{A}, \mu}^{(u)}(\boldsymbol{\xi} ; \alpha, \beta) \\
& = \begin{cases}\alpha+\sum_{1 \leq k \leq K} v_{a_{k}}^{\prime}\left(\xi_{j}\right)+\sum_{1 \leq l \leq L} v_{b_{l}}^{\prime}\left(\xi_{j}-\xi_{\ell}\right) & \text { if } j^{\prime}=j, \\
\sum_{1 \leq l \leq L} v_{b_{l}}^{\prime}\left(\xi_{j^{\prime}}-\xi_{j}\right) & \text { if } j^{\prime} \neq j,\end{cases}
\end{aligned}
$$

is manifestly positive definite:

$$
\begin{aligned}
& \sum_{1 \leq j, j^{\prime} \leq n} x_{j} x_{j^{\prime}} H_{j, j^{\prime}}^{\mathrm{A}}(\boldsymbol{\xi})= \\
& \sum_{1 \leq j \leq n}\left(\alpha+\sum_{1 \leq k \leq K} v_{a_{k}}^{\prime}\left(\xi_{j}\right)\right) x_{j}^{2}+\sum_{\substack{1 \leq j<j^{\prime} \leq n \\
1 \leq l \leq L}} v_{b_{l}}^{\prime}\left(\xi_{j}-\xi_{j^{\prime}}\right)\left(x_{j}-x_{j^{\prime}}\right)^{2}
\end{aligned}
$$

(since the derivatives $v_{a_{k}}^{\prime}(x)$ and $v_{b_{l}}^{\prime}(x)$ are positive for our parameter regime).

The upshot is that for any $\mu \in \Lambda_{\mathrm{A}}(2.4)$ the critical equation $\nabla_{\boldsymbol{\xi}} V_{\mathrm{A}, \mu}^{(u)}(\boldsymbol{\xi} ; \alpha, \beta)=0$, which is given explicitly by the following transcendental system

$$
\alpha \xi_{j}+\sum_{1 \leq k \leq K} v_{a_{k}}\left(\xi_{j}\right)+\sum_{\substack{1 \leq j^{\prime} \leq n, j^{\prime} \neq j \\ 1 \leq l \leq L}} v_{b_{l}}\left(\xi_{j}-\xi_{j^{\prime}}\right)=2 \pi\left(\mu_{j}+\beta\right)
$$

$(j=1, \ldots, n)$, has a unique solution $\boldsymbol{\xi}=\boldsymbol{\xi}_{\mathrm{A}, \mu}^{(u)}$ consisting of the global minimum of the strictly convex Morse function $V_{\mathrm{A}, \mu}^{(u)}(\boldsymbol{\xi} ; \alpha, \beta)(2.5)$.

Proposition 2.1 (Bethe roots of type A). (i) For the parameter regime in Eq. (2.3) and any $\mu \in \Lambda_{A}$ (2.4), the unique global minimum $\boldsymbol{\xi}=\boldsymbol{\xi}_{A, \mu}^{(u)}$ of $V_{A, \mu}^{(u)}(\boldsymbol{\xi} ; \alpha, \beta)$ (2.5) produces a solution of the Bethe system of type $A$ (2.2).

(ii) The assignment $\mu \rightarrow \boldsymbol{\xi}_{A, \mu}^{(u)}, \mu \in \Lambda_{A}$ is injective and depends smoothly on the parameters (2.3).

Proof. (i) Since for any $x \in \mathbb{R}$ and $a=a_{k}, b_{l}$ subject to the restrictions in Eq. (2.3):

$$
e^{-i v_{a}^{(u)}(x)}=\frac{\mathrm{s}^{(u)}(i a+x)}{\mathrm{S}^{(u)}(i a-x)}
$$

it is clear from Eq. (2.8) - upon multiplying by $i$ and exponentiating both sidesthat the critical point $\boldsymbol{\xi}=\boldsymbol{\xi}_{\mathrm{A}, \mu}^{(u)}$ solves the Bethe equations (2.2).

(ii) That the assignment $\mu \rightarrow \boldsymbol{\xi}_{\mathrm{A}, \mu}^{(u)}, \mu \in \Lambda_{\mathrm{A}}$ defines an injection is also immediate from the system in Eq. (2.8), while the smoothness in the parameters (2.3) follows from it by the implicit function theorem. Indeed, our system is manifestly smooth in these parameters and its Jacobian amounts to the Hessian (2.7) (and is thus invertible).

2.3. Bethe bounds. It follows from the system in Eq. (2.8) that the global minimum $\boldsymbol{\xi}_{\mathrm{A}, \mu}^{(u)}$ of $V_{\mathrm{A}, \mu}^{(u)}(\boldsymbol{\xi} ; \alpha, \beta)$ (2.5) with $\mu \in \Lambda_{\mathrm{A}}$ (2.4) belongs to the open wedge

$$
\mathbb{A}_{\mathrm{A}}:=\left\{\boldsymbol{\xi} \in \mathbb{R}^{n} \mid \xi_{1}>\xi_{2}>\cdots>\xi_{n}\right\} .
$$


Indeed, by subtracting the $j^{\prime}$ th equation from the $j$ th equation we see that

$$
\begin{aligned}
\alpha\left(\xi_{j}-\xi_{j^{\prime}}\right) & +\sum_{1 \leq k \leq K}\left(v_{a_{k}}\left(\xi_{j}\right)-v_{a_{k}}\left(\xi_{j^{\prime}}\right)\right) \\
& +\sum_{\substack{1 \leq j^{\prime \prime} \leq n \\
1 \leq l \leq L}}\left(v_{b_{l}}\left(\xi_{j}-\xi_{j^{\prime \prime}}\right)-v_{b_{l}}\left(\xi_{j^{\prime}}-\xi_{j^{\prime \prime}}\right)\right)=2 \pi\left(\mu_{j}-\mu_{j^{\prime}}\right) .
\end{aligned}
$$

Since $v_{a_{k}}(x)$ and $v_{b_{l}}(x)$ are monotonously increasing for our parameter regime, it is manifest from Eq. (2.11) that $\xi_{j}>\xi_{j^{\prime}}$ if $\mu_{j}>\mu_{j^{\prime}}$ (because otherwise the LHS would be $\leq 0$ while the RHS is $>0$ ). By refining this analysis somewhat further, one arrives at the following bounds on the gaps between $\xi_{j}$ and $\xi_{j^{\prime}}$ at $\boldsymbol{\xi}=\boldsymbol{\xi}_{\mathrm{A}, \mu}^{(u)}$.

Proposition 2.2 (Bethe bounds of type A). For the parameter regime in Eq. (2.3) and any $\mu \in \Lambda_{A}(2.4)$, one has that at the global minimum $\boldsymbol{\xi}=\boldsymbol{\xi}_{A, \mu}^{(u)}$ of $V_{A, \mu}^{(u)}(\boldsymbol{\xi} ; \alpha, \beta)$ (2.5):

$$
\frac{2 \pi\left(\mu_{j}-\mu_{j^{\prime}}\right)}{\alpha+\kappa_{-}^{(u)}} \leq \xi_{j}-\xi_{j^{\prime}} \leq \frac{2 \pi\left(\mu_{j}-\mu_{j^{\prime}}\right)}{\alpha+\kappa_{+}^{(u)}}
$$

for $1 \leq j<j^{\prime} \leq n$, where

$$
\kappa_{-}^{(u)}:= \begin{cases}2 \sum_{1 \leq k \leq K} a_{k}^{-1}+2 n \sum_{1 \leq l \leq L} b_{l}^{-1} & \text { if } u=r, \\ \sum_{1 \leq k \leq K} \cot \left(\frac{1}{2} a_{k}\right)+n \sum_{1 \leq l \leq L} \cot \left(\frac{1}{2} b_{l}\right) & \text { if } u=h, \\ \sum_{1 \leq k \leq K} \operatorname{coth}\left(\frac{1}{2} \operatorname{Re}\left(a_{k}\right)\right)+n \sum_{1 \leq l \leq L} \operatorname{coth}\left(\frac{1}{2} \operatorname{Re}\left(b_{l}\right)\right) & \text { if } u=t,\end{cases}
$$

and

$$
\kappa_{+}^{(u)}:= \begin{cases}0 & \text { if } u=r \text { or } u=h, \\ \sum_{1 \leq k \leq K} \tanh \left(\frac{1}{2} \operatorname{Re}\left(a_{k}\right)\right)+n \sum_{1 \leq l \leq L} \tanh \left(\frac{1}{2} \operatorname{Re}\left(b_{l}\right)\right) & \text { if } u=t .\end{cases}
$$

Proof. When $a=a_{k}$ or $b_{l}$ from Eq. (2.3), the derivative of $v_{a}(x)=v_{a}^{(u)}(x)$ (2.6) remains bounded:

$$
v_{a}^{\prime}(x) \in \begin{cases}{\left[0, \frac{2}{a}\right]} & \text { if } u=r, \\ {\left[0, \cot \left(\frac{a}{2}\right)\right]} & \text { if } u=h, \\ {\left[\tanh \left(\frac{1}{2} \operatorname{Re}(a)\right), \operatorname{coth}\left(\frac{1}{2} \operatorname{Re}(a)\right)\right]} & \text { if } u=t\end{cases}
$$

(for any $x \in \mathbb{R}$ ). Hence, if $1 \leq j<j^{\prime} \leq n$ (so $\mu_{j}>\mu_{j^{\prime}}$ and $\xi_{j}>\xi_{j^{\prime}}$ ) Eq. (2.11) implies (via the mean value theorem) that

$$
\left(\alpha+\kappa_{+}^{(u)}\right)\left(\xi_{j}-\xi_{j^{\prime}}\right) \leq 2 \pi\left(\mu_{j}-\mu_{j^{\prime}}\right) \leq\left(\alpha+\kappa_{-}^{(u)}\right)\left(\xi_{j}-\xi_{j^{\prime}}\right) .
$$

\section{Convex Bethe systems of type B}

In the case of quantum particle models with open-end boundary conditions rather than the more conventional periodic boundary conditions, the form of the Bethe Ansatz equations is known to undergo some structural modifications G71, G14, S88. Here we refer to the latter Bethe Ansatz equations as systems of type B, and we will again restrict attention to a relatively wide class of equations in the convex regime. The mathematics of trigonometric Bethe systems of type B was addressed in Refs. [LR04] (cf. also [05, Ch. 16.5]) and [B06] through q-difference Sturm-Liouville theory and $q$-deformed Onsager algebras, respectively. Specific examples of particle models leading to rational Bethe Ansatz equations of type B are 
the open quantum nonlinear Schrödinger equation [G71, G14, DE17] and its lattice discretization [DFR08]. The trigonometric/hyperbolic type B systems arise in turn as Bethe Ansatz equations for the $q$-boson model with open-end boundary interactions LW12, DE17, DEZ18. The open Heisenberg XXX and XXZ spin chains with boundary interactions ABBBQ87, S88, CLSW03, N04, MRM05, FSW08, BCR13. again lead to rational and trigonometric/hyperbolic Bethe Ansatz equations of type $\mathrm{B}$ that do not belong to the convex regime considered here, and thus fall outside the scope of the results presented below.

3.1. Bethe equations. The Bethe system of type B is defined as

$$
e^{2 i \alpha \xi_{j}}=(-1)^{\varepsilon} \prod_{1 \leq k \leq K} \frac{\mathrm{s}\left(i a_{k}+\xi_{j}\right)}{\mathrm{s}\left(i a_{k}-\xi_{j}\right)} \prod_{\substack{1 \leq j^{\prime} \leq n, j^{\prime} \neq j \\ 1 \leq l \leq L}} \frac{\mathrm{s}\left(i b_{l}+\xi_{j}+\xi_{j^{\prime}}\right)}{\mathrm{s}\left(i b_{l}-\xi_{j}-\xi_{j^{\prime}}\right)} \frac{\mathrm{s}\left(i b_{l}+\xi_{j}-\xi_{j^{\prime}}\right)}{\mathrm{s}\left(i b_{l}-\xi_{j}+\xi_{j^{\prime}}\right)}
$$

$(j=1, \ldots, n)$. Here $\mathrm{s}(x)=\mathrm{s}^{(u)}(x)$ is taken from Eq. 2.1), and in order to ensure the convexity of the system we will again impose the following restrictions on the $L+K+2$ parameters $\alpha, \varepsilon, a_{1}, \ldots, a_{K}$ and $b_{1}, \ldots, b_{L}$ :

$$
\alpha \in(0, \infty), \quad \varepsilon \in\{0,1\}, \quad a_{k}, b_{l} \in\left\{\begin{array}{ll|}
(0, \infty) & \text { if } u=r \\
(0, \pi) & \text { if } u=h \\
(0, \infty) \cup((0, \infty)+i \pi) & \text { if } u=t
\end{array}\right.
$$

$(k=1, \ldots, K, l=1, \ldots, L)$.

3.2. Bethe roots. Following the same pattern as for type A, we establish the Morse function whose equation for the critical points reproduces the Bethe system of type B (after exponentiation):

$$
\begin{aligned}
V_{\mathrm{B}, \mu}^{(u)}(\boldsymbol{\xi} ; \alpha, \varepsilon):= & \sum_{1 \leq j \leq n}\left(\alpha \xi_{j}^{2}-2 \pi\left(\mu_{j}+\frac{\varepsilon}{2}\right) \xi_{j}+\sum_{1 \leq k \leq K} \int_{0}^{\xi_{j}} v_{a_{k}}(x) \mathrm{d} x\right) \\
& +\sum_{\substack{1 \leq j \leq j^{\prime} \leq n \\
1 \leq l \leq L}}\left(\int_{0}^{\xi_{j}+\xi_{j^{\prime}}} v_{b_{l}}(x) \mathrm{d} x+\int_{0}^{\xi_{j}-\xi_{j^{\prime}}} v_{b_{l}}(x) \mathrm{d} x\right)
\end{aligned}
$$

with $v_{a}(x)=v_{a}^{(u)}(x)$ as in Subsection 2.2 and

$$
\mu \in \Lambda_{\mathrm{B}}:=\left\{\mu \in \mathbb{Z}^{n} \mid \mu_{1}>\mu_{2}>\cdots>\mu_{n}>0\right\} .
$$

As before, the parameter restrictions in Eq. (3.2) guarantee that (i) $V_{\mathrm{B}, \mu}^{(u)}(\boldsymbol{\xi} ; \alpha)$ is smooth in $\boldsymbol{\xi} \in \mathbb{R}^{n}$, (ii) $V_{\mathrm{B}, \mu}^{(u)}(\boldsymbol{\xi} ; \alpha) \rightarrow \infty$ when $|\boldsymbol{\xi}| \rightarrow \infty$, and (iii) $V_{\mathrm{B}, \mu}^{(u)}(\boldsymbol{\xi} ; \alpha)$ is strictly convex:

$$
\begin{aligned}
& H_{j, j^{\prime}}^{\mathrm{B}}(\boldsymbol{\xi}):=\partial_{\xi_{j}} \partial_{\xi_{j^{\prime}}} V_{\mathrm{B}, \mu}^{(u)}(\boldsymbol{\xi} ; \alpha, \varepsilon) \\
& = \begin{cases}2 \alpha+\sum_{1 \leq k \leq K} v_{a_{k}}^{\prime}\left(\xi_{j}\right)+\sum_{\substack{\ell \leq j \\
1 \leq l \leq L}}\left(v_{b_{l}}^{\prime}\left(\xi_{j}+\xi_{\ell}\right)+v_{b_{l}}^{\prime}\left(\xi_{j}-\xi_{\ell}\right)\right) & \text { if } j^{\prime}=j, \\
\sum_{1 \leq l \leq L}\left(v_{b_{l}}^{\prime}\left(\xi_{j^{\prime}}+\xi_{j}\right)+v_{b_{l}}^{\prime}\left(\xi_{j^{\prime}}-\xi_{j}\right)\right) & \text { if } j^{\prime} \neq j,\end{cases}
\end{aligned}
$$


SO

$$
\begin{aligned}
\sum_{1 \leq j, j^{\prime} \leq n} x_{j} x_{j^{\prime}} H_{j, j^{\prime}}^{\mathrm{B}}(\boldsymbol{\xi}) & =\sum_{1 \leq j \leq n}\left(2 \alpha+\sum_{1 \leq k \leq K} v_{a_{k}}^{\prime}\left(\xi_{j}\right)\right) x_{j}^{2} \\
& +\sum_{\substack{1 \leq j<j^{\prime} \leq n \\
1 \leq l \leq L}}\left(v_{b_{l}}^{\prime}\left(\xi_{j}+\xi_{j^{\prime}}\right)\left(x_{j}+x_{j^{\prime}}\right)^{2}+v_{b_{l}}^{\prime}\left(\xi_{j}-\xi_{j^{\prime}}\right)\left(x_{j}-x_{j^{\prime}}\right)^{2}\right) .
\end{aligned}
$$

Hence, for any $\mu \in \Lambda_{\mathrm{B}}(3.4)$ the critical equation $\nabla_{\boldsymbol{\xi}} V_{\mathrm{B}, \mu}^{(u)}(\boldsymbol{\xi} ; \alpha, \varepsilon)=0$, i.e. the system

$$
2 \alpha \xi_{j}+\sum_{1 \leq k \leq K} v_{a_{k}}\left(\xi_{j}\right)+\sum_{\substack{1 \leq j^{\prime} \leq n, j^{\prime} \neq j \\ 1 \leq l \leq L}}\left(v_{b_{l}}\left(\xi_{j^{\prime}}+\xi_{j}\right)-v_{b_{l}}\left(\xi_{j^{\prime}}-\xi_{j}\right)\right)=2 \pi\left(\mu_{j}+\frac{\varepsilon}{2}\right)
$$

$(j=1, \ldots, n)$, has a unique solution $\boldsymbol{\xi}=\boldsymbol{\xi}_{\mathrm{B}, \mu}^{(u)}$ given by the global minimum of the strictly convex Morse function $V_{\mathrm{B}, \mu}^{(u)}(\boldsymbol{\xi} ; \alpha, \varepsilon)$ (3.3).

Proposition 3.1 (Bethe roots of type B). (i) For the parameter regime in Eq. (3.2) and any $\mu \in \Lambda_{B}$ (3.4), the unique global minimum $\boldsymbol{\xi}=\boldsymbol{\xi}_{B, \mu}^{(u)}$ of $V_{B, \mu}^{(u)}(\boldsymbol{\xi} ; \alpha, \varepsilon)$ (3.3) produces a solution of the Bethe system of type $B$ (3.1).

(ii) The assignment $\mu \rightarrow \boldsymbol{\xi}_{B, \mu}^{(u)}, \mu \in \Lambda_{B}$ is injective and depends smoothly on the parameters (3.2).

Proof. The proof of Proposition 2.1 applies verbatim upon substituting: $\mathrm{A} \rightarrow \mathrm{B}$ and Eqs. (2.2), (2.3), (2.5), (2.7), (2.8) $\rightarrow$ Eqs. (3.1), (3.2), (3.3), (3.5), (3.6), respectively.

3.3. Bethe bounds. In the same way as in Section 2, we deduce from the system in Eq. (3.6) that the global minimum $\boldsymbol{\xi}_{\mathrm{B}, \mu}^{(u)}$ of $V_{\mathrm{B}, \mu}^{(u)}(\boldsymbol{\xi} ; \alpha, \varepsilon)$ (3.3) with $\mu \in \Lambda_{\mathrm{B}}$ (3.4) belongs to the open cone

$$
\mathbb{A}_{\mathrm{B}}:=\left\{\boldsymbol{\xi} \in \mathbb{R}^{n} \mid \xi_{1}>\xi_{2}>\cdots>\xi_{n}>0\right\} .
$$

Indeed, it is manifest from Eq. (3.6) that $\xi_{j}>0$ if $\mu_{j}>0$, because the expression on the LHS is monotonously increasing and odd in $\xi_{j}$ (for our parameter regime). Moreover, subtracting the $j^{\prime}$ th equation from the $j$ th equation now yields that

$$
\begin{aligned}
2 \alpha\left(\xi_{j}-\xi_{j^{\prime}}\right) & +\sum_{1 \leq k \leq K}\left(v_{a_{k}}\left(\xi_{j}\right)-v_{a_{k}}\left(\xi_{j^{\prime}}\right)\right) \\
& +\sum_{\substack{1 \leq j^{\prime \prime} \leq n, j^{\prime \prime} \neq j, j^{\prime} \\
1 \leq l \leq L}}\left(v_{b_{l}}\left(\xi_{j}+\xi_{j^{\prime \prime}}\right)-v_{b_{l}}\left(\xi_{j^{\prime}}+\xi_{j^{\prime \prime}}\right)\right) \\
& +\sum_{\substack{1 \leq j^{\prime \prime} \leq n \\
1 \leq l \leq L}}\left(v_{b_{l}}\left(\xi_{j}-\xi_{j^{\prime \prime}}\right)-v_{b_{l}}\left(\xi_{j^{\prime}}-\xi_{j^{\prime \prime}}\right)\right)=2 \pi\left(\mu_{j}-\mu_{j^{\prime}}\right),
\end{aligned}
$$

so by monotonicity $\xi_{j}>\xi_{j^{\prime}}$ if $\mu_{j}>\mu_{j^{\prime}}$ as before. Refining the analysis finally leads us to the following bounds on $\xi_{j}$ and on the gaps between $\xi_{j}$ and $\xi_{j^{\prime}}$ at $\boldsymbol{\xi}=\boldsymbol{\xi}_{\mathrm{B}, \mu}^{(u)}$. 
Proposition 3.2 (Bethe bounds of type B). For the parameter regime in Eq. (3.2) and any $\mu \in \Lambda_{B}(\underline{3.4})$, one has that at the global minimum $\boldsymbol{\xi}=\boldsymbol{\xi}_{B, \mu}^{(u)}$ of $V_{B, \mu}^{(u)}(\boldsymbol{\xi} ; \alpha, \varepsilon)$ (3.3):

$$
\frac{\pi\left(\mu_{j}+\frac{\varepsilon}{2}\right)}{\alpha+\kappa_{-}^{(u)}} \leq \xi_{j} \leq \frac{\pi\left(\mu_{j}+\frac{\varepsilon}{2}\right)}{\alpha+\kappa_{+}^{(u)}}
$$

for $1 \leq j \leq n$, and

$$
\frac{\pi\left(\mu_{j}-\mu_{j^{\prime}}\right)}{\alpha+\kappa_{-}^{(u)}} \leq \xi_{j}-\xi_{j^{\prime}} \leq \frac{\pi\left(\mu_{j}-\mu_{j^{\prime}}\right)}{\alpha+\kappa_{+}^{(u)}}
$$

for $1 \leq j<j^{\prime} \leq n$, where

$$
\kappa_{-}^{(u)}:= \begin{cases}\sum_{1 \leq k \leq K} a_{k}^{-1}+2(n-1) \sum_{1 \leq l \leq L} b_{l}^{-1} & \text { if } u=r, \\ \frac{1}{2} \sum_{1 \leq k \leq K} \cot \left(\frac{1}{2} a_{k}\right)+(n-1) \sum_{1 \leq l \leq L} \cot \left(\frac{1}{2} b_{l}\right) & \text { if } u=h, \\ \frac{1}{2} \sum_{1 \leq k \leq K} \operatorname{coth}\left(\frac{1}{2} \operatorname{Re}\left(a_{k}\right)\right)+(n-1) \sum_{1 \leq l \leq L} \operatorname{coth}\left(\frac{1}{2} \operatorname{Re}\left(b_{l}\right)\right) & \text { if } u=t,\end{cases}
$$

and

$$
\kappa_{+}^{(u)}:= \begin{cases}0 & \text { if } u=r \text { or } u=h, \\ \frac{1}{2} \sum_{1 \leq k \leq K} \tanh \left(\frac{1}{2} \operatorname{Re}\left(a_{k}\right)\right) & \\ +(n-1) \sum_{1 \leq l \leq L} \tanh \left(\frac{1}{2} \operatorname{Re}\left(b_{l}\right)\right) & \text { if } u=t .\end{cases}
$$

Proof. As in the proof of Proposition 3.2. one deduces - from the bounds on the derivative of $v_{a}^{(u)}(2.6)$ in combination the mean value theorem - via Eq. (3.6) that

$$
\left(\alpha+\kappa_{+}^{(u)}\right) \xi_{j} \leq \pi\left(\mu_{j}+\frac{\varepsilon}{2}\right) \leq\left(\alpha+\kappa_{-}^{(u)}\right) \xi_{j}
$$

for $1 \leq j \leq n$, and via Eq. (3.8) that

$$
\left(\alpha+\kappa_{+}^{(u)}\right)\left(\xi_{j}-\xi_{j^{\prime}}\right) \leq \pi\left(\mu_{j}-\mu_{j^{\prime}}\right) \leq\left(\alpha+\kappa_{-}^{(u)}\right)\left(\xi_{j}-\xi_{j^{\prime}}\right)
$$

for $1 \leq j<j^{\prime} \leq n$.

Remark 3.3. For $a$ complex and $x$ real, one has that

$$
\operatorname{Re}\left(v_{a}^{\prime}(x)\right)=\frac{1}{2}\left(v_{\operatorname{Re}(a)}^{\prime}(x+\operatorname{Im}(a))+v_{\operatorname{Re}(a)}^{\prime}(x-\operatorname{Im}(a))\right) .
$$

The upshot is that the propositions in Sections 2 and 3 persist for complex parameters $a_{k}$ and $b_{l}$, provided both all non-real parameters $a_{k}$ and all non-real parameters $b_{l}$ arise in complex conjugate pairs. Indeed, we may in this situation relax the parameter restrictions in Eqs. (2.3) and (3.2) by replacing $a_{k}$ with $\operatorname{Re}\left(a_{k}\right)$ and $b_{l}$ with $\operatorname{Re}\left(b_{l}\right)$, while performing the same modifications in the expressions for the bounds in Propositions 2.2 and 3.2 (when $u=r$ and $u=h$ ).

\section{Estimates for the zeros of Askey-Wilson and Wilson polynomials}

The estimates for the zeros of the (Askey-)Wilson polynomials hinge on algebraic Bethe equations that arise from the type B system in Eqs. (3.1), (3.2) via the degeneration $\alpha \rightarrow 0$. For this purpose, it is enough to restrict attention to the case that $\varepsilon=0$, which will therefore be assumed from now on (unless explicitly stated otherwise). 
4.1. Algebraic Bethe system of type $\mathbf{B}$ at $\alpha=0$. The following proposition adapts the results of Section 3 for $u=r$ and $u=t$ to the case $\alpha=0$ with $\mu \in \Lambda_{B}$ (3.4) minimal:

$$
\mu=\rho:=(n, n-1, n-2, \ldots, 2,1)
$$

(and $\varepsilon=0$ ).

Proposition 4.1 (Bethe system of type B at $\alpha=0$ ). Let $K>2, L>0$, and let $a_{1}, \ldots, a_{K}$ and $b_{1}, \ldots, b_{L}$ satisfy the restrictions in Eq. (3.2). For $u=r$ and $u=t$ the unique global minimum $\boldsymbol{\xi}_{B, \rho}^{(u)}$ of the strictly convex Morse function $V_{B, \rho}^{(u)}(\boldsymbol{\xi} ; 0,0)$ (3.3), (4.1) produces a solution to the algebraic Bethe system (3.1) at $\alpha=0$ and $\varepsilon=$ 0 , which depends smoothly on the parameters $a_{1}, \ldots, a_{K}$ and $b_{1}, \ldots, b_{L}$. Moreover, at $\boldsymbol{\xi}=\boldsymbol{\xi}_{B, \rho}^{(u)}$ the following inequalities are satisfied:

$$
\frac{\pi(n+1-j)}{\kappa_{-}^{(r)}} \leq \xi_{j}
$$

$(1 \leq j \leq n)$ and

$$
\frac{\pi\left(j^{\prime}-j\right)}{\kappa_{-}^{(r)}} \leq \xi_{j}-\xi_{j^{\prime}}
$$

$\left(1 \leq j<j^{\prime} \leq n\right)$ if $u=r$, and

$$
\frac{\pi(n+1-j)}{\kappa_{-}^{(t)}} \leq \xi_{j} \leq \frac{\pi(n+1-j)}{\kappa_{+}^{(t)}}
$$

$(1 \leq j \leq n)$ and

$$
\frac{\pi\left(j^{\prime}-j\right)}{\kappa_{-}^{(t)}} \leq \xi_{j}-\xi_{j^{\prime}} \leq \frac{\pi\left(j^{\prime}-j\right)}{\kappa_{+}^{(t)}}
$$

$\left(1 \leq j<j^{\prime} \leq n\right)$ if $u=t$, with $\kappa_{ \pm}^{(u)}$ taken from Proposition 3.2.

Proof. We only need to check the existence of the unique global minimum of the Morse function at $(\alpha, \varepsilon)=(0,0)$, since the rest of the proof can be extracted verbatim from the proofs of Propositions 3.1 and 3.2 via the specialization $\mu=\rho$, $\alpha=0$, and $\varepsilon=0$. It is moreover clear from the Hessian (3.5) that the Morse function $V_{\mathrm{B}, \mu}^{(u)}(\boldsymbol{\xi} ; 0,0)$ (3.3) remains strictly convex if $K>0$, which automatically settles the question of the minimum's uniqueness. For the existence to persist, it is enough to infer that $V_{\mathrm{B}, \rho}^{(u)}(\boldsymbol{\xi} ; 0,0) \rightarrow \infty$ for $|\boldsymbol{\xi}| \rightarrow \infty$.

When $u=t$ the quadratic growth of the integral $\int_{0}^{\xi} v_{a}^{(t)}(x) \mathrm{d} x$ as $|\xi| \rightarrow \infty-$ which stems from the quasi-periodicity $v_{a}^{(t)}(x+2 \pi)=v_{a}^{(t)}(x)+2 \pi$-immediately guarantees the desired growth of $V_{\mathrm{B}, \mu}^{(t)}(\boldsymbol{\xi} ; 0,0)$ for $K>0$, therewith confirming the existence of the (unique) global minimum of our Morse function for any $\mu \in \Lambda_{B}$ in this situation.

When $u=r$ one has that $\lim _{x \rightarrow \infty} v_{a}^{(r)}(x)=\pi$. This case is therefore more subtle, as the existence of the global minimum of $V_{\mathrm{B}, \mu}^{(r)}(\boldsymbol{\xi} ; 0,0)$ is no longer guaranteed for all $\mu \in \Lambda_{B}$ (3.4). We notice though that - apart from the linear term-our Morse function is symmetric with respect to the natural action of the hyperoctahedral group of signed permutations on the components of $\boldsymbol{\xi}$ (because $\int_{0}^{\xi} v_{a}^{(r)}(x) \mathrm{d} x$ is even in $\xi$ ). It thus suffices to infer that $V_{\mathrm{B}, \rho}^{(r)}(\boldsymbol{\xi} ; 0,0) \rightarrow \infty$ when $|\boldsymbol{\xi}| \rightarrow \infty$ on the closure 
of the fundamental cone $\mathbb{A}_{\mathrm{B}}$ (3.7). Notice in this connection also that the linear term $\rho_{1} \xi_{1}+\cdots+\rho_{n} \xi_{n}$ grows fastest for $|\boldsymbol{\xi}| \rightarrow \infty$ on this fundamental cone, because

$$
\rho_{1}\left(\xi_{1}-\epsilon_{1} \xi_{\sigma_{1}}\right)+\cdots+\rho_{n}\left(\xi_{n}-\epsilon_{n} \xi_{\sigma_{n}}\right) \geq 0
$$

for all $\boldsymbol{\xi} \in \mathbb{A}_{\mathrm{B}}$ (3.7), $\left\{\sigma_{1}, \ldots, \sigma_{n}\right\}=\{1, \ldots, n\}$ and $\epsilon_{j} \in\{1,-1\}(j=1, \ldots, n)$.

To verify the unbounded growth of $V_{\mathrm{B}, \rho}^{(r)}(\boldsymbol{\xi} ; 0,0)$ on the closure of $\mathbb{A}_{\mathrm{B}}$, we set $x_{j}=\xi_{j}-\xi_{j+1}(j=1, \ldots, n)$ with the convention that $\xi_{n+1} \equiv 0$. Moreover, for a given nonempty subset $J \subset\{1, \ldots, n\}$, let us write $\boldsymbol{\xi} \stackrel{J}{\rightarrow} \infty$ if $x_{j} \rightarrow \infty$ for $j \in J$ while $x_{j}$ remains bounded for $j \notin J$. Since

$$
\xi_{j}=x_{j}+\cdots+x_{n}
$$

$(1 \leq j \leq n)$ and

$$
\begin{aligned}
& \xi_{j}-\xi_{j^{\prime}}=x_{j}+\cdots+x_{j^{\prime}-1}, \\
& \xi_{j}+\xi_{j^{\prime}}=x_{j}+\cdots+x_{j^{\prime}-1}+2\left(x_{j^{\prime}}+\cdots+x_{n}\right)
\end{aligned}
$$

$\left(1 \leq j<j^{\prime} \leq n\right)$, it follows that for $\boldsymbol{\xi} \stackrel{J}{\rightarrow} \infty$

$$
\begin{aligned}
\sum_{1 \leq j \leq n} \int_{0}^{\xi_{j}} v_{a_{k}}^{(r)}(x) \mathrm{d} x & \sim \pi \sum_{j \in J} j x_{j}, \\
\sum_{1 \leq j<j^{\prime} \leq n} \int_{0}^{\xi_{j}-\xi_{j^{\prime}}} v_{b_{l}}^{(r)}(x) \mathrm{d} x & \sim \pi \sum_{j \in J} j(n-j) x_{j}, \\
\sum_{1 \leq j<j^{\prime} \leq n} \int_{0}^{\xi_{j}+\xi_{j^{\prime}}} v_{b_{l}}^{(r)}(x) \mathrm{d} x & \sim \pi \sum_{j \in J} j(n-1) x_{j} .
\end{aligned}
$$

Hence, the leading asymptotics of $V_{\mathrm{B}, \rho}^{(r)}(\boldsymbol{\xi} ; 0,0)$ for $\boldsymbol{\xi} \stackrel{J}{\rightarrow} \infty$ is given by

$$
\pi \sum_{j \in J} j x_{j}(-(2 n+1-j)+K+L(2 n-1-j)) \geq \pi \sum_{j \in J} j x_{j}
$$

(where for the last estimate it was used that $K>2$ and $L>0$ ). By varying our choice for $J$, this diverging lower bound on the leading asymptotics confirms that $V_{\mathrm{B}, \rho}^{(r)}(\boldsymbol{\xi} ; 0,0) \rightarrow \infty$ for $|\boldsymbol{\xi}| \rightarrow \infty$ (first on the closure of the fundamental cone $\mathbb{A}_{\mathrm{B}}$, and then on the whole $\mathbb{R}^{n}$ because of the hyperoctahedral symmetry of the nonlinear terms and Eq. (4.4)).

Remark 4.2. In the hyperbolic case one has that $\lim _{x \rightarrow \infty} v_{a}^{(h)}(x)=\pi-a$ (with $0<a<\pi$ ), which entails that the existence of the (unique) global minimum of $V_{\mathrm{B}, \rho}^{(h)}(\boldsymbol{\xi} ; 0,0)$ is only guaranteed for $K>2$ and $L>0$ provided the parameters $a_{k}$ and $b_{l}$ lie sufficiently close to 0 in the interval $(0, \pi)$.

Remark 4.3. It is clear (from the stated inequalities) that the global minimum $\boldsymbol{\xi}=\boldsymbol{\xi}_{\mathrm{B}, \rho}^{(u)}$ of $V_{\mathrm{B}, \rho}^{(u)}(\boldsymbol{\xi} ; 0,0)$ in Proposition 4.1 is again assumed inside the cone $\mathbb{A}_{\mathrm{B}}$ (3.7), both for $u=r$ and $u=t$. In the latter (trigonometric) case, the Bethe roots under consideration belong in fact to the interval $(0, \pi)$, i.e. one has that

$$
\pi>\xi_{1}>\xi_{2}>\cdots>\xi_{n}>0
$$

at the critical point of $V_{\mathrm{B}, \rho}^{(t)}(\boldsymbol{\xi} ; 0,0)$. Indeed, for $u=t$ the LHS of Eq. (3.6) takes the value $(2 \alpha+K+2 L(n-1)) \pi$ at $\xi_{j}=\pi$ (by the quasi-periodicity $v_{a}^{(t)}(x+2 \pi)=$ 
$v_{a}^{(t)}(x)+2 \pi$ of the odd function $v_{a}^{(t)}(x)(2.6)$ ), while the RHS takes only the value $2 \pi\left(n+1-j+\frac{\epsilon}{2}\right)$ when $\mu=\rho$ (4.1). Hence, it is immediate from the monotonicity of the LHS as function of $\xi_{j}$ that the corresponding Bethe solutions must be smaller than $\pi$ when $K>2$ and $L>0$.

4.2. Wilson polynomials. The Wilson polynomial W80, [KLS10, Ch. 9.1]

$$
\begin{aligned}
\mathrm{p}_{n}(\xi ; a, b, c, d):= & \frac{(-1)^{n}(a+b, a+c, a+d)_{n}}{(n+a+b+c+d-1)_{n}} \\
& \times{ }_{4} F_{3}\left[\begin{array}{c}
-n, n+a+b+c+d-1, a+i \xi, a-i \xi \\
a+b, a+c, a+d
\end{array}\right]
\end{aligned}
$$

is a monic polynomial of degree $n$ in $\xi^{2}$ that satisfies the second-order difference equation

$$
A(\xi)\left(\mathrm{p}_{n}(\xi+i)-\mathrm{p}_{n}(\xi)\right)+A(-\xi)\left(\mathrm{p}_{n}(\xi-i)-\mathrm{p}_{n}(\xi)\right)=E_{n} \mathrm{p}_{n}(\xi)
$$

with

$$
\begin{aligned}
A(\xi) & =\frac{(\xi+i a)(\xi+i b)(\xi+i c)(\xi+i d)}{2 \xi(2 \xi+i)}, \\
E_{n} & =-n(n+a+b+c+d-1)
\end{aligned}
$$

(as a rational identity in the parameters $a, b, c, d$ ). For $a, b, c, d>0$ the Wilson polynomials $\mathrm{p}_{n}(\xi ; a, b, c, d), n=0,1,2, \ldots$ are (manifestly) analytic in the parameters and constitute an orthogonal basis on the interval $(0, \infty)$ with respect to the weight function

$$
\Delta(\xi)=\left|\frac{\Gamma(a+i \xi) \Gamma(b+i \xi) \Gamma(c+i \xi) \Gamma(d+i \xi)}{\Gamma(2 i \xi)}\right|^{2}
$$

(where $\Gamma(\cdot)$ refers to the gamma function).

Remark 4.4. It is helpful to view the difference equation (4.7) as an eigenvalue equation with eigenvalue $E_{n}$ for Wilson's second-order difference operator $D$ acting on $\mathrm{p}_{n}(\xi)$ at the LHS. The fact that the monic Wilson polynomials $\mathrm{p}_{n}(\xi ; a, b, c, d)$, $n=0,1,2, \ldots$ solve the eigenvalue equations in question implies that $D$ preserves the space of even polynomials in $\xi$ without raising the degree. Since the corresponding eigenvalues $E_{n}$ are nondegenerate for $a, b, c, d>0$, this triangularity of the Wilson operator with respect to the monomial basis $\xi^{2 n}, n=0,1,2, \ldots$ guarantees that its eigenbasis is unique in the space of even polynomials (up to normalization):

$$
\mathrm{p}_{n}(\xi ; a, b, c, d)=\left(\prod_{0 \leq m<n} \frac{D-E_{m}}{E_{n}-E_{m}}\right) \xi^{2 n}, \quad n=0,1,2, \ldots
$$

(using, e.g., the Cayley-Hamilton theorem in the finite-dimensional invariant subspace of polynomials of degree at most $n$ in $\xi^{2}$ ).

Theorem 4.5 (Zeros of the Wilson polynomials). For $a, b, c, d>0$, the zeros $\xi_{1}^{(n)}>\xi_{2}^{(n)}>\cdots>\xi_{n}^{(n)}>0$ of the Wilson polynomial $\mathrm{p}_{n}(\xi ; a, b, c, d)$ (4.6) obey the following inequalities:

$$
\frac{\pi(n+1-j)}{k_{-}^{(n)}(a, b, c, d)} \leq \xi_{j}^{(n)}
$$


$(1 \leq j \leq n)$ and

$$
\frac{\pi\left(j^{\prime}-j\right)}{k_{-}^{(n)}(a, b, c, d)} \leq \xi_{j}^{(n)}-\xi_{j^{\prime}}^{(n)}
$$

$\left(1 \leq j<j^{\prime} \leq n\right)$, where

$$
k_{-}^{(n)}(a, b, c, d):=2(n-1)+a^{-1}+b^{-1}+c^{-1}+d^{-1} .
$$

Proof. Let $\boldsymbol{\xi}=\boldsymbol{\xi}_{\mathrm{B}, \rho}^{(r)}$ denote the global minimum of $V_{\mathrm{B}, \rho}^{(r)}(\boldsymbol{\xi} ; 0,0)$ (3.3), (4.1) for $K=4$ and $L=1$, with

$$
a_{1}=a, a_{2}=b, a_{3}=c, a_{4}=d \quad \text { and } \quad b_{1}=1 .
$$

Following OS04, D05, SYZ09, BC14, we shall check that the associated polynomial

$$
\mathrm{p}_{n}(\xi)=\left(\xi^{2}-\xi_{1}^{2}\right)\left(\xi^{2}-\xi_{2}^{2}\right) \cdots\left(\xi^{2}-\xi_{n}^{2}\right)
$$

satisfies the difference equation (4.7). To this end we first observe that the substitution of $\mathrm{p}_{n}(\xi)$ into Eq. (4.7) gives rise to a polynomial relation of degree $n$ in $\xi^{2}$ (cf. Remark 4.4). Since $\xi_{1}>\xi_{2}>\cdots>\xi_{n}>0$ (cf. Remark 4.3), it is sufficient to infer the polynomial relation in question at $\xi=\xi_{j}(j=1, \ldots, n)$. This entails the following algebraic relations

$$
A\left(\xi_{j}\right) \mathrm{p}_{n}\left(\xi_{j}+i\right)+A\left(-\xi_{j}\right) \mathrm{p}_{n}\left(\xi_{j}-i\right)=0 \quad(j=1, \ldots, n),
$$

which in turn hold by Proposition 4.1. Indeed-upon making $A(\cdot)$ and $\mathrm{p}_{n}(\cdot)$ explicit - it is readily seen that Eq. (4.13) amounts precisely to the $K=4, L=1$ Bethe system of type B with parameters (4.11) that is solved by $\boldsymbol{\xi}=\boldsymbol{\xi}_{\mathrm{B}, \rho}^{(r)}$.

The upshot is that $\mathrm{p}_{n}(\xi ; a, b, c, d)$ (4.6) and $\mathrm{p}_{n}(\xi)$ (4.12) are both monic polynomials of degree $n$ in $\xi^{2}$ satisfying the eigenvalue equation (4.7). Since the corresponding eigenvalues $E_{n}, n=0,1,2, \ldots$ are nondegenerate for $a, b, c, d>0$, this implies (cf. Remark 4.4) that $\mathrm{p}_{n}(\xi ; a, b, c, d)=\mathrm{p}_{n}(\xi)$, i.e. $\xi_{j}^{(n)}=\xi_{j}(j=1, \ldots, n)$. The asserted inequalities for the zeros are now inherited from those for $\boldsymbol{\xi}=\boldsymbol{\xi}_{\mathrm{B}, \rho}^{(r)}$ in Proposition 4.1

4.3. Askey-Wilson polynomials. The Askey-Wilson polynomial AW85, KLS10, Ch. 14.1]

$$
\begin{aligned}
\mathrm{p}_{n}(\xi ; a, b, c, d ; q):= & \frac{(a b, a c, a d ; q)_{n}}{(2 a)^{n}\left(a b c d q^{n-1} ; q\right)_{n}} \\
& \times{ }_{4} \Phi_{3}\left[\begin{array}{c}
q^{-n}, a b c d q^{n-1}, a e^{i \xi}, a e^{-i \xi} \\
a b, a c, a d
\end{array} ; q, q\right]
\end{aligned}
$$

is a monic polynomial of degree $n$ in $\cos (\xi)$. It satisfies the second-order difference equation

$$
A(\xi)\left(\mathrm{p}_{n}(\xi-i \log (q))-\mathrm{p}_{n}(\xi)\right)+A(-\xi)\left(\mathrm{p}_{n}(\xi+i \log (q))-\mathrm{p}_{n}(\xi)\right)=E_{n} \mathrm{p}_{n}(\xi)
$$

with

$$
\begin{aligned}
A(\xi) & =\frac{\left(1-a e^{i \xi}\right)\left(1-b e^{i \xi}\right)\left(1-c e^{i \xi}\right)\left(1-d e^{i \xi}\right)}{\left(1-e^{2 i \xi}\right)\left(1-q e^{2 i \xi}\right)} \\
E_{n} & =q^{-n}\left(1-q^{n}\right)\left(1-a b c d q^{n-1}\right)
\end{aligned}
$$


(as a rational identity in the parameters $a, b, c, d$ and $q$ ). For $-1<a, b, c, d, q<1$, the Askey-Wilson polynomials are analytic in the parameters and constitute an orthogonal basis on the interval $(0, \pi)$ with respect to the weight function

$$
\Delta(\xi)=\left|\frac{\left(e^{2 i \xi} ; q\right)_{\infty}}{\left(a e^{i \xi}, b e^{i \xi}, c e^{i \xi}, d e^{i \xi} ; q\right)_{\infty}}\right|^{2} .
$$

Notice that this means in particular that the singularities of $\mathrm{p}_{n}(\xi ; a, b, c, d ; q)$ (4.14) at $a=0$ and $q=0$ are in fact removable.

Theorem 4.6 (Zeros of the Askey-Wilson polynomials). For $-1<a, b, c, d, q<$ 1 , the zeros $\pi>\xi_{1}^{(n)}>\xi_{2}^{(n)}>\cdots>\xi_{n}^{(n)}>0$ of the Askey-Wilson polynomial $\mathrm{p}_{n}(\xi ; a, b, c, d ; q)$ (4.14) obey the following inequalities:

$$
\frac{\pi(n+1-j)}{k_{-}^{(n)}(a, b, c, d ; q)} \leq \xi_{j}^{(n)} \leq \frac{\pi(n+1-j)}{k_{+}^{(n)}(a, b, c, d ; q)}
$$

$(1 \leq j \leq n)$ and

$$
\frac{\pi\left(j^{\prime}-j\right)}{k_{-}^{(n)}(a, b, c, d ; q)} \leq \xi_{j}^{(n)}-\xi_{j^{\prime}}^{(n)} \leq \frac{\pi\left(j^{\prime}-j\right)}{k_{+}^{(n)}(a, b, c, d ; q)}
$$

$\left(1 \leq j<j^{\prime} \leq n\right)$, where

$$
\begin{aligned}
& k_{ \pm}^{(n)}(a, b, c, d ; q):=(n-1)\left(\frac{1-|q|}{1+|q|}\right)^{ \pm 1}+ \\
& \quad \frac{1}{2}\left(\left(\frac{1-|a|}{1+|a|}\right)^{ \pm 1}+\left(\frac{1-|b|}{1+|b|}\right)^{ \pm 1}+\left(\frac{1-|c|}{1+|c|}\right)^{ \pm 1}+\left(\frac{1-|d|}{1+|d|}\right)^{ \pm 1}\right) .
\end{aligned}
$$

Proof. Let $\boldsymbol{\xi}=\boldsymbol{\xi}_{\mathrm{B}, \rho}^{(t)}$ denote the global minimum of $V_{\mathrm{B}, \rho}^{(t)}(\boldsymbol{\xi} ; 0,0)$ (3.3), (4.1) for $K=4$ and $L=1$, with

$$
e^{-a_{1}}=a, e^{-a_{2}}=b, e^{-a_{3}}=c, e^{-a_{4}}=d \quad \text { and } \quad e^{-b_{1}}=q
$$

(where it is temporarily assumed that $a b c d q \neq 0$ ). With the aid of D05] (cf. also [LR04, I05. Ch. 16.5], and OS05, SYZ09, BC16), the proof of Proposition 4.5) is readily adapted to the Askey-Wilson level starting from trigonometric polynomial

$$
\mathrm{p}_{n}(\xi)=\left(\cos (\xi)-\cos \left(\xi_{1}\right)\right)\left(\cos (\xi)-\cos \left(\xi_{2}\right)\right) \cdots\left(\cos (\xi)-\cos \left(\xi_{n}\right)\right) .
$$

Indeed, (similarly as before) the fact that $\mathrm{p}_{n}(\xi)$ (4.19) solves the second-order difference equation (4.15) encodes a trigonometric identity that is equivalent to the following algebraic relations between the nodes $\pi>\xi_{1}>\xi_{2}>\cdots>\xi_{1}>0$ :

$$
A\left(\xi_{j}\right) \mathrm{p}_{n}\left(\xi_{j}-i \log (q)\right)+A\left(-\xi_{j}\right) \mathrm{p}_{n}\left(\xi_{j}+i \log (q)\right)=0 \quad(j=1, \ldots, n) .
$$

Moreover, after making $A(\cdot)$ and $\mathrm{p}_{n}(\cdot)$ explicit and invoking of the elementary trigonometric relation $\cos (\xi)-\cos \left(\xi_{j}\right)=2 \sin \left(\frac{1}{2}\left(\xi_{j}+\xi\right)\right) \sin \left(\frac{1}{2}\left(\xi_{j}-\xi\right)\right)$, the identities in Eq. (4.20) become a manifest consequence of Proposition 4.1 upon identification with the trigonometric Bethe system of type B solved by $\boldsymbol{\xi}=\boldsymbol{\xi}_{\mathrm{B}, \rho}^{(t)}$.

Since for $a, b, c, d, q \in(-1,1) \backslash\{0\}$ the eigenvalues $E_{n}$ stemming from Eq. (4.15) are again nondegenerate, it is deduced through the difference equation that $\mathrm{p}_{n}(\xi ; a, b, c, d ; q)=\mathrm{p}_{n}(\xi)$, and thus $\xi_{j}^{(n)}=\xi_{j}(j=1, \ldots, n)$. (Here one uses again 
that the Askey-Wilson difference operator acting on the LHS of Eq. 4.15) is triangular on the monomial basis $\cos ^{n}(\xi), n=0,1,2, \ldots$, cf. Remark 4.4) The asserted inequalities for the zeros now follow in the same manner as before from the formulas in Proposition 4.1 through specialization. Finally, the resulting inequalities are readily extended to the case of one or more vanishing parameters $a, b, c, d, q$ by continuity.

\section{Estimates FOR THE ZeROS OF THE SYMmetric CONTINUOUS HAHN POLYNOMIALS}

After performing the degeneration $\alpha \rightarrow 0$, the rational Bethe system of type A in Eqs. (2.2), (2.3) entails lower bounds for the zeros of the symmetric continuous Hahn polynomials.

5.1. Rational Bethe system of type $\mathbf{A}$ at $\alpha=0$. The following proposition adapts the results of Section 2 for $u=r$ to the case $\alpha=0$ with $\mu \in \Lambda_{A}$ (2.4) chosen minimal:

$$
\mu=\tilde{\rho}:=\left(\left\lfloor\frac{n-1}{2}\right\rfloor,\left\lfloor\frac{n-3}{2}\right\rfloor,\left\lfloor\frac{n-5}{2}\right\rfloor, \ldots,\left\lfloor\frac{3-n}{2}\right\rfloor,\left\lfloor\frac{1-n}{2}\right\rfloor\right)
$$

and

$$
\beta=\beta_{n}:= \begin{cases}\frac{1}{2} & \text { if } n=\text { even } \\ 0 & \text { if } n=\text { odd }\end{cases}
$$

Proposition 5.1 (Rational Bethe system of type A at $\alpha=0$ ). Let $K, L>0$ and let $a_{1}, \ldots, a_{K}$ and $b_{1}, \ldots, b_{L}$ satisfy the restrictions in $E q$. (2.3). The unique global minimum $\boldsymbol{\xi}_{A, \tilde{\rho}}^{(r)}$ of the strictly convex Morse function $V_{A, \tilde{\rho}}^{(r)}\left(\boldsymbol{\xi} ; 0, \beta_{n}\right)$ (2.5), (5.1a), (5.1b) produces a solution to the algebraic Bethe system (2.2) at $\alpha=0$, which depends smoothly on the parameters $a_{1}, \ldots, a_{K}$ and $b_{1}, \ldots, b_{L}$. Moreover, at $\boldsymbol{\xi}=$ $\boldsymbol{\xi}_{A, \tilde{\rho}}^{(r)}$ the following inequalities are satisfied:

$$
\frac{2 \pi\left(j^{\prime}-j\right)}{\kappa_{-}^{(r)}} \leq \xi_{j}-\xi_{j^{\prime}}
$$

$\left(1 \leq j<j^{\prime} \leq n\right)$, with $\kappa_{-}^{(r)}$ taken from Proposition 2.2.

Proof. The statements are derived by adapting the proof of Proposition 4.1 to the present context. Like before, it suffices to concentrate on the existence and the uniqueness of the global minimum of the Morse function at $\alpha=0$, as all other arguments can be extracted verbatim from the proofs of Propositions 2.1 and 2.2 via the specialization $\mu=\tilde{\rho}, \alpha=0$ and $\beta=\beta_{n}$. Since for $K \geq 1$ the strict convexity at $\alpha=0$ is manifest from the Hessian (2.7), we will only infer the existence of the global minimum by verifying that the Morse function $V_{\mathrm{A}, \tilde{\rho}}^{(r)}\left(\boldsymbol{\xi} ; 0, \beta_{n}\right) \rightarrow \infty$ for $|\boldsymbol{\xi}| \rightarrow \infty$. Upon exploiting the permutation symmetry of the nonlinear part of the Morse function, the required asymptotic analysis is restricted to the closure of the fundamental wedge $\mathbb{A}_{\mathrm{A}}(2.10)$, where we also use that the growth of the linear term $\left(\tilde{\rho}_{1}+\beta_{n}\right) \xi_{1}+\cdots+\left(\tilde{\rho}_{n}+\beta_{n}\right) \xi_{n}$ is maximal on this wedge because of the inequality

$$
\left(\tilde{\rho}_{1}+\beta_{n}\right)\left(\xi_{1}-\xi_{\sigma_{1}}\right)+\cdots+\left(\tilde{\rho}_{n}+\beta_{n}\right)\left(\xi_{n}-\xi_{\sigma_{n}}\right) \geq 0
$$

for all $\boldsymbol{\xi} \in \mathbb{A}_{\mathrm{A}}$ and $\left\{\sigma_{1}, \ldots, \sigma_{n}\right\}=\{1, \ldots, n\}$.

To verify the unbounded growth of $V_{\mathrm{A}, \tilde{\rho}}^{(r)}\left(\boldsymbol{\xi} ; 0, \beta_{n}\right)$ on the closure of $\mathbb{A}_{\mathrm{A}}$, we again set $x_{j}=\xi_{j}-\xi_{j+1}(j=1, \ldots, n)$ with the convention that $\xi_{n+1} \equiv 0$. Given a 
nonempty subset $J \subset\{1, \ldots, n\}$, let us now write $\boldsymbol{\xi} \stackrel{J}{\rightarrow} \infty$ if $x_{j} \rightarrow \infty$ for $j \in J$ with $j<n,\left|x_{n}\right| \rightarrow \infty$ for $n \in J$, while $x_{j}$ remains bounded for $j \notin J$. The leading asymptotics of $V_{\mathrm{A}, \tilde{\rho}}^{(r)}\left(\boldsymbol{\xi} ; 0, \beta_{n}\right)$ for $\boldsymbol{\xi} \stackrel{J}{\rightarrow} \infty$ is then readily verified to dominate

$$
\pi \sum_{j \in J} j x_{j}(-(n-j)+L(n-j))+ \begin{cases}\pi K \sum_{j \in J} j x_{j} & \text { if } n \notin J \\ \pi K\left|x_{n}\right| & \text { if } n \in J\end{cases}
$$

By varying our choice for $J$ (and assuming $K, L>0$ ), this diverging lower bound on the leading asymptotics confirms that $V_{\mathrm{A}, \rho}^{(r)}\left(\boldsymbol{\xi} ; 0, \beta_{n}\right) \rightarrow \infty$ when $|\boldsymbol{\xi}| \rightarrow \infty$ (first on the closure of the fundamental wedge $\mathbb{A}_{\mathrm{A}}$, and then on the whole $\mathbb{R}^{n}$ because of the permutation symmetry of the nonlinear terms and Eq. (5.3) ).

5.2. Symmetric continuous Hahn polynomials. The symmetric continuous Hahn polynomial [AW82, KLS10, Ch. 9.4]

$$
\begin{aligned}
\mathrm{p}_{n}(\xi ; a, b):= & \frac{i^{n}(2 a, a+b)_{n}}{(n+2 a+2 b-1)_{n}} \\
& \times{ }_{3} F_{2}\left[\begin{array}{c}
-n, n+2 a+2 b-1, a+i \xi \\
2 a, a+b
\end{array}\right]
\end{aligned}
$$

defines a monic polynomial of degree $n$ in $\xi$ satisfying the second-order difference equation

$$
A(\xi)\left(\mathrm{p}_{n}(\xi+i)-\mathrm{p}_{n}(\xi)\right)+A(-\xi)\left(\mathrm{p}_{n}(\xi-i)-\mathrm{p}_{n}(\xi)\right)=E_{n} \mathrm{p}_{n}(\xi)
$$

with

$$
\begin{aligned}
A(\xi) & =(\xi+i a)(\xi+i b), \\
E_{n} & =-n(n+2 a+2 b-1)
\end{aligned}
$$

(as a rational identity in the parameters $a, b$ ). For $a, b>0$ the continuous Hahn polynomials $\mathrm{p}_{n}(\xi ; a, b), n=0,1,2, \ldots$ are (manifestly) analytic in the parameters and form an orthogonal basis on $\mathbb{R}$ with respect to the weight function

$$
\Delta(\xi)=|\Gamma(a+i \xi) \Gamma(b+i \xi)|^{2} .
$$

Theorem 5.2 (Zeros of the symmetric continuous Hahn polynomials). For $a, b>0$, the zeros $\xi_{1}^{(n)}>\xi_{2}^{(n)}>\cdots>\xi_{n}^{(n)}$ of the symmetric continuous Hahn polynomial $\mathrm{p}_{n}(\xi ; a, b)$ (5.4) obey the following inequalities:

$$
\frac{\pi\left(j^{\prime}-j\right)}{k_{-}^{(n)}(a, b)} \leq \xi_{j}^{(n)}-\xi_{j^{\prime}}^{(n)}
$$

$\left(1 \leq j<j^{\prime} \leq n\right)$, where

$$
k_{-}^{(n)}(a, b):=n+a^{-1}+b^{-1}
$$

Proof. The proof is very similar as that for the Wilson polynomials in Theorem 5.2. Let $\boldsymbol{\xi}=\boldsymbol{\xi}_{\mathrm{A}, \tilde{\rho}}^{(r)}$ denote the global minimum of $V_{\mathrm{A}, \tilde{\rho}}^{(r)}\left(\boldsymbol{\xi} ; 0, \beta_{n}\right)$ (2.5), (5.1a), (5.1b) for $K=2$ and $L=1$, with

$$
a_{1}=a, a_{2}=b \quad \text { and } \quad b_{1}=1 .
$$

Following OS04, SYZ09, we now infer that the associated polynomial

$$
\mathrm{p}_{n}(\xi)=\left(\xi-\xi_{1}\right)\left(\xi-\xi_{2}\right) \cdots\left(\xi-\xi_{n}\right)
$$


satisfies the difference equation (5.5). Indeed, (as before) substitution of $\mathrm{p}_{n}(\xi)$ into Eq. (5.5) and inferring the corresponding polynomial relation of degree $n$ in $\xi$ at the nodes $\xi_{1}>\xi_{2}>\cdots>\xi_{n}$ gives rise to the algebraic relations

$$
A\left(\xi_{j}\right) \mathrm{p}_{n}\left(\xi_{j}+i\right)+A\left(-\xi_{j}\right) \mathrm{p}_{n}\left(\xi_{j}-i\right)=0 \quad(j=1, \ldots, n) .
$$

When making $A(\cdot)$ and $\mathrm{p}_{n}(\cdot)$ explicit, Eq. (5.10) (and thus Eq. (5.5)) is seen to hold upon identification with the $K=2, L=1$ Bethe system of type A with parameters (5.8) solved by $\boldsymbol{\xi}=\boldsymbol{\xi}_{\mathrm{A}, \tilde{\rho}}^{(r)}$.

In view of the triangularity of the action of the pertinent continuous Hahn difference operator on the monomial basis $\xi^{n}, n=0,1,2, \ldots$ (cf. Remark 4.4), the monic polynomials $\mathrm{p}_{n}(\xi ; a, b)$ (5.4) and $\mathrm{p}_{n}(\xi)$ (5.9) thus coincide, as solutions of the eigenvalue equation (5.5) corresponding to the same nondegenerate eigenvalue $E_{n}$. The upshot is that $\xi_{j}^{(n)}=\xi_{j}(j=1, \ldots, n)$, so the asserted inequalities for the zeros are again inherited from those for $\boldsymbol{\xi}=\boldsymbol{\xi}_{\mathrm{A}, \tilde{\rho}}^{(r)}$ stemming from Proposition 5.1 .

Remark 5.3. Because the weight function $\Delta(\xi)(5.6)$ is even in $\xi$, the symmetric continuous Hahn polynomial $p_{n}(\xi ; a, b)$ (5.4) is even or odd in $\xi$ depending whether $n$ is even or odd, respectively. Its zeros are therefore positioned symmetrically around the origin: $\xi_{n+1-j}^{(n)}=-\xi_{j}^{(n)}(j=1, \ldots, n)$. For $a, b>0$, the following lower bounds for the positive zeros of $p_{n}(\xi ; a, b)$ (5.4) are now an immediate consequence of Theorem 5.2 (upon picking $j^{\prime}=n+1-j$ ):

$$
\frac{\pi(n+1-2 j)}{2 k_{-}^{(n)}(a, b)} \leq \xi_{j}^{(n)} \quad \text { for } j=1, \ldots,\left\lfloor\frac{n}{2}\right\rfloor .
$$

Remark 5.4. It follows from the proofs of Theorems 4.5, 4.6 and 5.2 that the vector of decreasingly ordered zeros $\boldsymbol{\xi}^{(n)}:=\left(\xi_{1}^{(n)}, \ldots, \xi_{n}^{(n)}\right)$ of the Wilson, Askey-Wilson and symmetric continuous Hahn polynomials minimize $V_{\mathrm{B}, \rho}^{(r)}(\boldsymbol{\xi} ; 0,0)$ and $V_{\mathrm{B}, \rho}^{(t)}(\boldsymbol{\xi} ; 0,0)$ for $K=4, L=1$, and $V_{\mathrm{A}, \tilde{\rho}}^{(r)}\left(\boldsymbol{\xi} ; 0, \beta_{n}\right)$ for $K=2, L=1$, where the parameters are taken from Eqs. (4.11), (4.18) and (5.8), respectively. A different interpretation for these zeros as the minimizers of corresponding $n$-particle Ruijsenaars-Schneider type Hamiltonians from Refs. [D94, D95] has been pointed out in Refs. OS04, D05, OS05.

Remark 5.5. In view of Remark 3.3, the inequalities in Theorem 4.6 persist in the case that $a, b, c, d$ become complex within the open unit disc with non-real parameters arising in complex conjugate pairs. Similarly, Theorem 4.5 and Theorem 5.2 extend to the situation that $\operatorname{Re}(a), \operatorname{Re}(b), \operatorname{Re}(c), \operatorname{Re}(d)>0$ and $\operatorname{Re}(a), \operatorname{Re}(b)>0$ with non-real parameters occurring in complex conjugate pairs, upon replacing $k_{-}^{(n)}(a, b, c, d)$ by $k_{-}^{(n)}(\operatorname{Re}(a), \operatorname{Re}(b), \operatorname{Re}(c), \operatorname{Re}(d))$ and $k_{-}^{(n)}(a, b)$ by $k_{-}^{(n)}(\operatorname{Re}(a), \operatorname{Re}(b))$, respectively.

Remark 5.6. The following small numerical sample illustrates that the bounds stemming from Theorem 4.6 estimate the positions of the first few roots of the AskeyWilson polynomials reasonably well for parameter values $a, b, c, d, q$ not far from 0 . Notice, however, that for the large(st) root(s) our upper bound soon becomes trivial $(>\pi)$ even in a small example. The lower bounds for the roots of the Wilson polynomials and of the continuous Hahn polynomials originating from Theorem 4.5 
TABLE 1. Roots and their bounds for the Askey-Wilson polynomial $\mathrm{p}_{n}(\xi ; a, b, c, d ; q)$ (4.14) with $n=5, a=0.300, b=-0.200$, $c=0.150, d=0.100$, and $q=0.100$.

\begin{tabular}{|c|ccccc|}
\hline$n=5$ & $\xi_{5}^{(n)}$ & $\xi_{4}^{(n)}$ & $\xi_{3}^{(n)}$ & $\xi_{2}^{(n)}$ & $\xi_{1}^{(n)}$ \\
\hline Root & 0.496 & 0.997 & 1.508 & 2.033 & $2.577 \mid$ \\
\hline Lower bound & 0.400 & 0.800 & 1.200 & 1.600 & 2.000 \\
\hline Upper bound & 0.675 & 1.350 & 2.025 & 2.700 & 3.375 \\
\hline
\end{tabular}

TABLE 2. Roots and their lower bounds for the Wilson polynomial $\mathrm{p}_{n}(\xi ; a, b, c, d)$ (4.6) with $n=5, a=1.150, b=1.100, c=1.000$, and $d=0.900$.

\begin{tabular}{|c|ccccc|}
\hline$n=5$ & $\xi_{5}^{(n)}$ & $\xi_{4}^{(n)}$ & $\xi_{3}^{(n)}$ & $\xi_{2}^{(n)}$ & $\xi_{1}^{(n)}$ \\
\hline Root & 0.632 & 1.292 & 2.090 & 3.099 & 4.477 \\
\hline Lower bound & 0.264 & 0.528 & 0.793 & 1.057 & 1.321 \\
\hline
\end{tabular}

TABLE 3. Positive roots and their lower bounds for the symmetric continuous Hahn polynomial $\mathrm{p}_{n}(\xi ; a, b)$ (5.4) with $n=10, a=$ 1.100 and $b=0.900$.

\begin{tabular}{|c|ccccc|}
\hline$n=10$ & $\xi_{5}^{(n)}$ & $\xi_{4}^{(n)}$ & $\xi_{3}^{(n)}$ & $\xi_{2}^{(n)}$ & $\xi_{1}^{(n)}$ \\
\hline Root & 0.261 & 0.838 & 1.554 & 2.481 & 3.770 \\
\hline Lower bound & 0.131 & 0.392 & 0.653 & 0.915 & 1.176 \\
\hline
\end{tabular}

and from Theorem 5.2 (cf. also Remark 5.3) tend to be less sharp than in the Askey-Wilson case.

\section{ACKNOWLEDGMENTS}

Thanks are due to the referees for pointing out some improvements of the presentation.

\section{REFERENCES}

ALM82. S. Ahmed, A. Laforgia, and M.E. Muldoon, On the spacing of the zeros of some classical orthogonal polynomials, J. London Math. Soc. (2) 25 (1982), 246-252.

ABBBQ87. F.C. Alcaraz, M.N. Barber, M.T. Batchelor, R.J. Baxter, and G.R.W. Quispel, Surface exponents of the quantum XXZ, Ashkin-Teller and Potts models, J. Phys. A 20 (1987), 6397-6409.

ADGR12. I. Area, D.K. Dimitrov, E. Godoy, and F.R. Rafaeli, Inequalities for zeros of Jacobi polynomials via Obrechkoff's theorem, Math. Comp. 81 (2012), 991-1004.

ADGP13. I. Area, D.K. Dimitrov, E. Godoy, and V. Paschoa, Zeros of classical orthogonal polynomials of a discrete variable, Math. Comp. 82 (2013), 1069-1095.

AW82. R. Askey and J. Wilson, A set of hypergeometric orthogonal polynomials, SIAM J. Math. Anal. 13 (1982), 651-655. 
AW85. R. Askey and J. Wilson, Some basic hypergeometric orthogonal polynomials that generalize Jacobi polynomials, Mem. Amer. Math. Soc. 54 (1985), no. 319, 55 pp.

B06. P. Baseilhac, The $q$-deformed analogue of the Onsager algebra: beyond the Bethe ansatz approach, Nuclear Phys. B 754 (2006), 309-328.

B82. R.J. Baxter, Exactly solved models in statistical mechanics, Academic Press, Inc., London, 1982.

BCR13. S. Belliard, N. Crampé, and E. Ragoucy, Algebraic Bethe ansatz for open XXX model with triangular boundary matrices, Lett. Math. Phys. 103 (2013), 493-506.

BC14. O. Bihun and F. Calogero, Properties of the zeros of the polynomials belonging to the Askey scheme, Lett. Math. Phys. 104 (2014), 1571-1588.

BC16. O. Bihun and F. Calogero, Properties of the zeros of the polynomials belonging to the q-Askey scheme, J. Math. Anal. Appl. 433 (2016), no. 1, 525-542.

BB92. N.M. Bogoliubov and R.K. Bullough, A q-deformed completely integrable Bose gas model, J. Phys. A 25 (1992), 4057-4071.

BIK98. N.M. Bogoliubov, A.G. Izergin, and A.N. Kitanine, Correlation functions for a strongly correlated boson system, Nuclear Phys. B 516 (1998), 501-528.

CLSW03. J. Cao, H.-Q. Lin, K.-J. Shi, and Y. Wang, Exact solution of XXZ spin chain with unparallel boundary fields, Nucl. Phys. B 663 (2003), 487-519.

C87. L. Chihara, On the zeros of the Askey-Wilson polynomials, with applications to coding theory, SIAM J. Math. Anal. 18 (1987), 191-207.

DHK10. A. Deaño, D. Huybrechs, and A.B.J. Kuijlaars, Asymptotic zero distribution of complex orthogonal polynomials associated with Gaussian quadrature, J. Approx. Theory 162 (2010), 2202-2224.

D94. J.F. van Diejen, Integrability of difference Calogero-Moser systems, J. Math. Phys. 35 (1994), 2983-3004.

D95. J.F. van Diejen, Difference Calogero-Moser systems and finite Toda chains, J. Math. Phys. 36 (1995),1299-1323.

D05. J.F. van Diejen, On the equilibrium configuration of the $B C$-type RuijsenaarsSchneider system, J. Nonlinear Math. Phys. 12 (2005), suppl. 1, 689-696.

D06. J.F. van Diejen, Diagonalization of an integrable discretization of the repulsive delta Bose gas on the circle, Comm. Math. Phys. 267 (2006), 451-476.

DE17. J.F. van Diejen and E. Emsiz, Orthogonality of Bethe Ansatz eigenfunctions for the Laplacian on a hyperoctahedral Weyl alcove, Comm. Math. Phys. 350 (2017), 10171067.

DEZ18. J.F. van Diejen, E. Emsiz, and I.N. Zurrián, Completeness of the Bethe Ansatz for an open $q$-boson system with integrable boundary interactions, Ann. Henri Poincaré 19 (2018), 1349-1384.

D93. T.C. Dorlas, Orthogonality and completeness of the Bethe ansatz eigenstates of the nonlinear Schroedinger model, Comm. Math. Phys. 154 (1993), 347-376.

DFR08. A. Doikou, D. Fioravanti, F. Ravanini, The generalized non-linear Schrödinger model on the interval, Nuclear Physics B 790 (2008) 465-492.

D09. K. Driver, A note on the interlacing of zeros and orthogonality, J. Approx. Theory 161 (2009), 508-510.

DJ12. K. Driver and K. Jordaan, Bounds for extreme zeros of some classical orthogonal polynomials, J. Approx. Theory 164 (2012), 1200-1204.

ELR94. A. Elbert, A. Laforgia, and L.G. Rodon, On the zeros of Jacobi polynomials, Acta Math. Hungar. 64 (1994), 351-359.

FR86. P.J. Forrester and J.B. Rogers, Electrostatics and the zeros of the classical polynomials, SIAM J. Math. Anal. 17 (1986), 461-468.

FSW08. H. Frahm, A. Seel, and T. Wirth, Separation of variables in the open $X X X$ chain, Nuclear Phys. B 802 (2008), 351-367.

G87. L. Gatteschi, New inequalities for the zeros of Jacobi polynomials, SIAM J. Math. Anal. 18 (1987), 1549-1562.

G71. M. Gaudin, Boundary energy of a Bose gas in one dimension. Phys. Rev. A 4 (1971), 386-394.

G14. M. Gaudin, The Bethe Wavefunction, Cambridge University Press, Cambridge, 2014. 
G04. W. Gautschi, Orthogonal Polynomials: Computation and Approximation, Numerical Mathematics and Scientific Computation, Oxford Science Publications. Oxford University Press, New York, 2004.

GJRS16. P. Gochhayat, K. Jordaan, K. Raghavendar, and A. Swaminathan, Interlacing properties and bounds for zeros of ${ }_{2} \phi_{1}$ hypergeometric and little $q$-Jacobi polynomials, Ramanujan J. 40 (2016), 45-62.

G98. F.A. Grünbaum, Variations on a theme of Heine and Stieltjes: an electrostatic interpretation of the zeros of certain polynomials, J. Comput. Appl. Math. 99 (1998), 189-194.

GH96. F.A. Grünbaum and L. Haine, The $q$-version of a theorem of Bochner, J. Comput. Appl. Math. 68 (1996), 103-114.

HV12. M. Haneczok and W. Van Assche, Interlacing properties of zeros of multiple orthogonal polynomials, J. Math. Anal. Appl. 389 (2012), 429-438.

I00. M.E.H. Ismail, An electrostatics model for zeros of general orthogonal polynomials, Pacific J. Math. 193 (2000), 355-369.

I05. M.E.H. Ismail, Classical and Quantum Orthogonal Polynomials in One Variable, Encyclopedia of Mathematics and its Applications, vol. 98, Cambridge University Press, Cambridge, 2005.

ILR04. M.E.H. Ismail, S.S. Lin, and S.S. Roan, Bethe Ansatz Equations of XXZ Model and q-Sturm-Liouville Problems, arXiv:math-ph/0407033

IK81. A.G. Izergin and V.E. Korepin, A lattice model connected with a nonlinear Schrdinger equation, Dokl. Akad. Nauk SSSR 259 (1981), 76-79, arXiv:math.QA/0910.0295

JT09. K. Jordaan and F. Tookos, Convexity of the zeros of some orthogonal polynomials and related functions, J. Comput. Appl. Math. 233 (2009), 762-767.

KLS10. R. Koekoek, P.A. Lesky, and R. Swarttouw, Hypergeometric Orthogonal Polynomials and their q-Analogues, Springer Monographs in Mathematics. Springer-Verlag, Berlin, 2010.

KBI93. V.E. Korepin, N.M. Bogoliubov, and A.G. Izergin, Quantum Inverse Scattering Method and Correlation Functions, Cambridge University Press, Cambridge, 1993.

K13. C. Korff, Cylindric versions of specialised Macdonald functions and a deformed Verlinde algebra, Comm. Math. Phys, 318 (2013), 173-246.

K18. K.K. Kozlowski, On condensation properties of Bethe roots associated with the XXZ chain, Comm. Math. Phys, 357 (2018), 1009-1069.

KS13. K.K. Kozlowski and E.K. Sklyanin, Combinatorics of generalized Bethe equations, Lett. Math. Phys. 103 (2013), 1047-1077.

LW12. B. Li and Y.-S. Wang, Exact solving $q$ deformed boson model under open boundary condition, Modern Phys. Lett. B 26 (2012), 1150008.

LL63. E.H. Lieb and W. Liniger, Exact analysis of an interacting Bose gas. I. The general solution and the ground state, Phys. Rev. (2) 130 (1963), 1605-1616.

MMM07. F. Marcellán, A. Martínez-Finkelshtein, and P. Martínez-González, Electrostatic models for zeros of polynomials: old, new, and some open problems, J. Comput. Appl. Math. 207 (2007), 258-272.

M94. D.C. Mattis, The Many-Body Problem: An Encyclopedia of Exactly Solved Models in One Dimension, World Scientific, Singapore, 1994.

MRM05. C.S. Melo, G.A.P. Ribeiro, and M.J. Martins, Bethe ansatz for the XXX-S chain with non-diagonal open boundaries, Nucl. Phys. B 711 (2005), 565-603.

MTV09. E. Mukhin,V. Tarasov, and A, Varchenko, Bethe algebra of homogeneous XXX Heisenberg model has simple spectrum, Comm. Math. Phys. 288 (2009), 1-42.

M93. M.E. Muldoon, Properties of zeros of orthogonal polynomials and related functions, J. Comput. Appl. Math. 48 (1993), 167-186.

N04. R.I. Nepomechie, Bethe ansatz solution of the open XXZ chain with nondiagonal boundary terms, J. Phys. A 37 (2004), 433-440.

OS04. S. Odake and R. Sasaki, Equilibria of 'discrete' integrable systems and deformation of classical orthogonal polynomials, J. Phys. A 37 (2004), 11841-11876.

OS05. S. Odake and R. Sasaki, Equilibrium positions, shape invariance and Askey-Wilson polynomials, J. Math. Phys. 46 (2005), no. 6, 063513, 10 pp. 
BETHE ANSATZ AND ZEROS OF HYPERGEOMETRIC ORTHOGONAL POLYNOMIALS 21

SYZ09. R. Sasaki, W.-L.Yang, and Y.-Z. Zhang, Bethe ansatz solutions to quasi exactly solvable difference equations, SIGMA Symmetry Integrability Geom. Methods Appl. 5 (2009), Paper 104, 16 pp.

S16. B. Simanek, An electrostatic interpretation of the zeros of paraorthogonal polynomials on the unit circle, SIAM J. Math. Anal. 48 (2016), 2250-2268.

S10. B. Simon, Fine structure of the zeros of orthogonal polynomials: a progress report, Recent Trends in Orthogonal Polynomials and Approximation Theory, J.Arvesú, F. Marcellán and A. Martínez-Finkelshtein (eds.), Contemp. Math. 507, Amer. Math. Soc., Providence, RI, 2010, 241-254.

S88. E.K. Sklyanin, Boundary conditions for integrable quantum systems, J. Phys. A 21 (1988), 2375-2389.

S75. G. Szegö, Orthogonal Polynomials, Fourth Edition, American Mathematical Society, Colloquium Publications, vol. XXIII, American Mathematical Society, Providence, R.I., 1975.

T83. L.A. Takhtajan, Integrable models in classical and quantum field theory, Proceedings of the International Congress of Mathematicians, Vol. 1, 2 (Warsaw, 1983), Z. Ciesielski and C. Olech (eds.), North-Holland Publishing Co., Amsterdam, 1984, 1331-1346.

T06. N.V. Tsilevich, The quantum inverse scattering method for the $q$-boson model and symmetric functions, Funct. Anal. Appl. 40 (2006), 207-217.

W80. J.A. Wilson, Some hypergeometric orthogonal polynomials, SIAM J. Math. Anal. 11 (1980), 690-701.

YY69. C.N. Yang and C.P. Yang, Thermodynamics of a one-dimensional system of bosons with repulsive delta-function interaction, J. Math. Phys. 10 (1969), 1115-1122.

Instituto de Matemática y Física, Universidad de Talca, Casilla 747, Talca, Chile

E-mail address: diejen@inst-mat.utalca.cl

Facultad de Matemáticas, Pontificia Universidad Católica de Chile, Casilla 306 , Correo 22, Santiago, Chile

E-mail address: eemsiz@mat.uc.cl 